\title{
Burden of selected infectious diseases covered by UK childhood vaccinations: systematic literature review
}

\author{
James Clark-Wright*,1, Pollyanna Hudson², Conor McCloskey² \& Stuart Carroll ${ }^{1}$ \\ ${ }^{1}$ Sanofi Pasteur UK \& Ireland, Reading, UK \\ ${ }^{2}$ DRG Abacus, Bicester, Oxfordshire, UK \\ *Author for correspondence: Tel.: +44 0845023 0441; james.clark@alumni.oxon.net
}

\begin{abstract}
Aim: An overview of recent epidemiology and disease burden, independent of patient age, of diphtheria, tetanus, pertussis, hepatitis B, poliomyelitis and Hemophilus influenzae invasive disease in the UK. Materials \& methods: A systematic review was undertaken. Outcomes included incidence, prevalence, risk factors and cost burden. Results: 39 publications were included. Hepatitis B prevalence is high among certain risk groups. A small pertussis risk remains in pregnancy and for infants, which led to the introduction of maternal vaccination. $H$. influenzae invasive disease cases are limited to rare serotypes. Polio, tetanus and diphtheria are well controlled. Conclusion: The evaluated diseases are currently well controlled, thanks to a comprehensive vaccination program, with a generally low clinical and cost burden.
\end{abstract}

First draft submitted: 7 July 2020; Accepted for publication: 29 October 2020; Published online: 19 November 2020

Keywords: burden • diphtheria $\bullet$ Hemophilus influenzae invasive disease $\bullet$ hepatitis B • pertussis $\bullet$ poliomyelitis $\bullet$ tetanus $\bullet$ vaccine-preventable infectious diseases

Vaccination, especially in childhood, is recognized globally as one of the most important healthcare interventions [1]. In the UK, routine vaccination against diphtheria (D) was introduced in 1942 [2], drastically reducing the number of cases and deaths [3]. In the past 20 years, the number of diphtheria cases in the UK has been as low as approximately 10-20 cases per year [3]. Inactivated polio vaccine (IPV), pertussis (whole-cell pertussis vaccine [wP]), tetanus (T) and Hemophilus influenzae Type b (Hib) vaccines, were first introduced in 1956, 1957, 1961 and 1992, respectively. Together with diphtheria vaccine, they became part of a combined, pentavalent childhood vaccine (DTaP/IPV/Hib) from 2004 onwards [2]. At this point, wP was replaced by the acellular pertussis vaccine (aP) used in $\mathrm{DTaP} / \mathrm{IPV} / \mathrm{Hib}$ [4]. Compared with monovalent vaccines, combination vaccines have a number of potential benefits, including: decreased number of vaccinations (especially important in a crowded vaccination schedule), decreased pain/discomfort, fewer injection site reactions and increased compliance (resulting in higher coverage rates) [5]. Although combination vaccines can be more costly than the combined costs of individual vaccines, which can be attributed to the inherent manufacturing complexities of combining multiple antigens, adjuvants and excipients [5], their benefits generally result in cost savings [5]. In England and Wales, pertussis notifications reached approximately 170,000 annually before wP was included in the vaccination schedule [6], which subsequently led to a drastic reduction of reported cases in all age groups [4,6]. The replacement of wP with aP in 2004 was based on data showing that $\mathrm{aP}$ is at least as effective as wP but associated with fewer adverse events [7]. However, there are indications that the efficacy of aP may decline faster than that of ${ }_{\mathrm{wP}}[8]$. Before the introduction of the Hib vaccine in 1992, Hib was the most common cause of childhood meningitis [9]. The inclusion of Hib in the vaccine program led to a $98 \%$ decline in the incidence of invasive Hib infections [9], and there have been no deaths due to invasive Hib disease in children in England and Wales since 2011 [10]. The incidence of tetanus has also remained low and the majority of cases occurring in England are in older adults who have not received the recommended vaccination program [11]. Only seven cases of tetanus were reported in England and Wales in 2013, all of which were in individuals who were either partially immunized, unimmunized or had no vaccination history available [12]. IPV led to a rapid and efficient reduction in cases of poliomyelitis in the UK [13]. The last recorded case of poliomyelitis 
due to indigenous virus in the UK occurred in the mid-1970s [13]. Polio viruses are today close to extinction, with the only reservoirs remaining in two countries without sufficient vaccine coverage (Afghanistan and Pakistan) [14], Nigeria having been declared free of wild polio in August 2020. Migration from and travel to and from such destinations where vaccine coverage is poor may contribute to a risk of recurrence.

For the 2016-2017 period, the national coverage of the DTaP/IPV/Hib pentavalent childhood vaccination by a child's first birthday was $93.1 \%$ in England [15]. Although this proportion is high, the target of $95 \%$ has not been met, except on a local level, where 68 of the 149 local authorities met the 95\% target [16]. From 2017 in the UK the pentavalent childhood vaccination was replaced by a hexavalent version, which additionally incorporates a hepatitis B (Hep B) component [2]. The adoption of universal Hep B vaccination in the UK demonstrates the willingness of the UK authorities to prioritize public health and make improvements to the vaccination schedule in order to protect and improve the health of the general public.

The aim of this systematic review is to provide an overview of recent data around the epidemiology and disease burden, independent of patient age, of diphtheria, tetanus, pertussis, Hep B, poliomyelitis, and $H$. influenzae invasive disease in the UK, for which vaccinations are included in the UK childhood vaccination schedule at 2, 3 and 4 months of age.

\section{Materials \& methods}

The protocol for this systematic literature review was not registered in a public database but is held with DRG Abacus and Sanofi Pasteur. Inclusion criteria were aligned with the population, interventions, comparators, outcomes, and study design criteria. Detailed eligibility criteria are listed in Supplementary Table 1. As a brief overview, studies on diphtheria, tetanus, pertussis, Hep B, poliomyelitis and H. influenzae were included, with no restriction on intervention, comparator, study design, patient age or publication date. Outcomes included incidence, prevalence, risk factors and data on cost burden. Only English-language publications were included. MEDLINE, Embase and The Cochrane Library were searched electronically. In addition, the reference lists of identified publications were hand searched, as well as several conference proceedings and additional sources/websites (see Supplementary Material for details). The full search strategies used, text words (free text), subject index headings (e.g., MeSH) and the relationship between search terms (e.g., Boolean operators) are provided in Supplementary Table 2. The searches were performed on the 5th of September 2017. The selection of abstracts and full texts for inclusion was performed by a single reviewer. The relevance of each citation identified was initially based on the title and abstract according to the selection criteria. For each included abstract, full text publications were obtained and evaluated based on all predefined selection criteria. Inclusion and exclusion of citations was verified by a second reviewer and disputes as to eligibility were referred to a third party for arbitration. Relevant data from included studies were extracted into predesigned data extraction tables in Microsoft ${ }^{\circledR}$ Excel. The extracted data were verified by a second reviewer according to the study protocol and any disputes were again referred to a third party. A quality (risk of bias) assessment was conducted using the adapted method of Drummond and Jefferson [17], as recommended by the National Institute for Health and Care Excellence.

\section{Results}

In total, 11,451 publications were identified through the electronic searches. Upon removal of 1288 duplicates, 10,163 titles and abstracts were available for screening. Of the 308 potentially relevant publications reviewed in full, 91 were excluded. Hand searching yielded a further seven relevant studies, resulting in 224 included publications. Due to this high number of publications, and given the focus of our work on the most recent evidence, only studies published in or after 2012 as full publications were included in a post hoc selection, which resulted in 39 eligible publications [11,18-54] (reporting on 38 studies) (Figure 1). Of these, 21 studies (22 publications) reported data on Hep B [20,21,23,25,26,31-34,36,38-46,48,49,52], nine on pertussis [6,18,22,24,30,50,51,53,54], five on $H$. influenzae [27-29,35,37] and one on poliomyelitis [47], tetanus [11], and diphtheria [19], respectively. Characteristics of the included studies can be found in Supplementary Tables 3-5.

\section{Hepatitis B}

Based on 2014 data from the British Pediatric Surveillance Unit (UK and Ireland), the annual incidence of acute infectious hepatitis is $0.52 / 100,000$ in children $<14$ years old; $4 \%$ of these cases were attributed to Hep B infection [20]. Capture-recapture analysis of diagnosed cases of chronic hepatitis B among children in England between 2001 and 2009 resulted in a prevalence estimate of 4.6/100,000 children <15 years old [36]. Of 325 


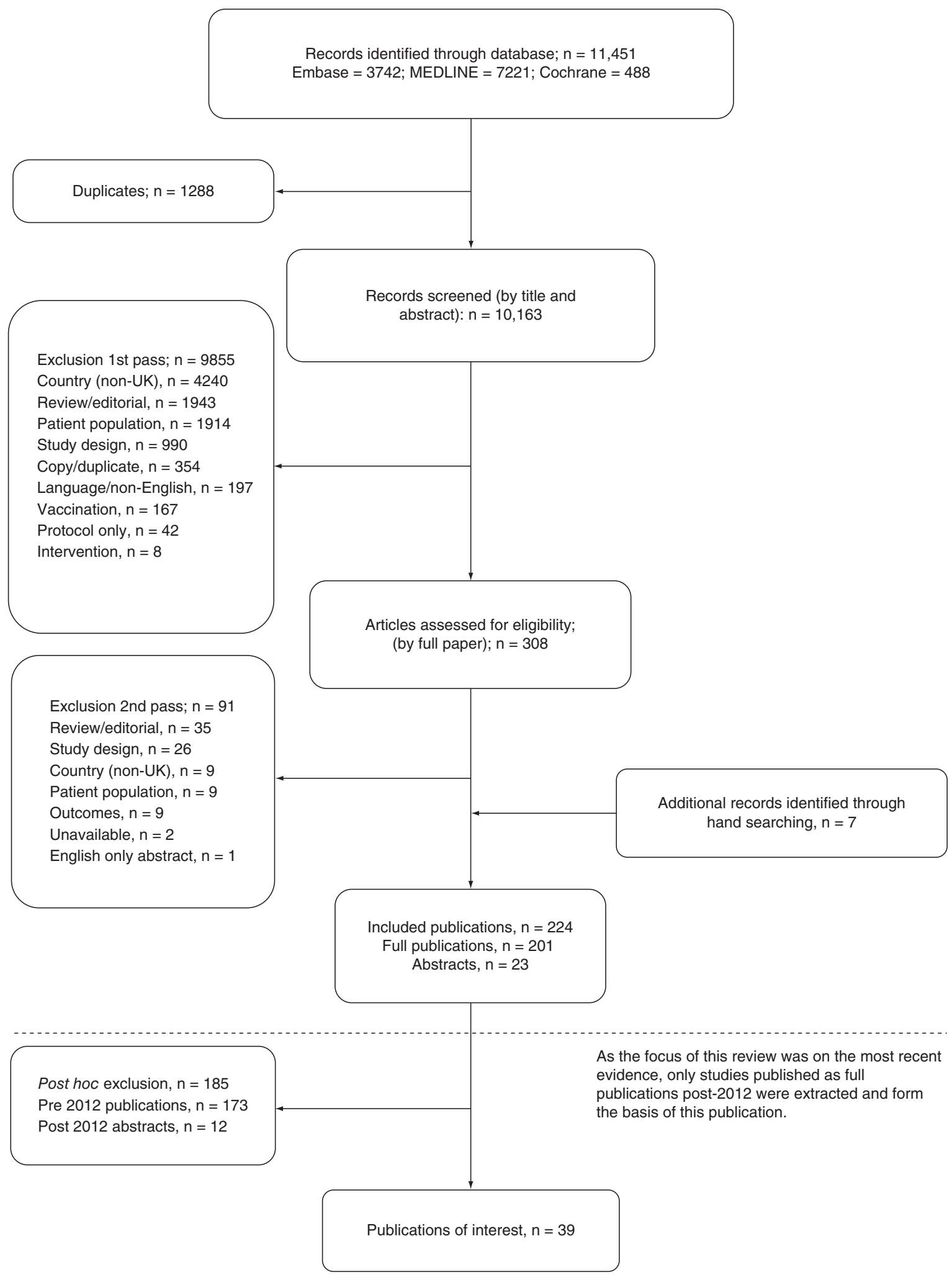

Figure 1. PRISMA flow diagram. 
pediatric cases under specialist care, 50\% had been born overseas, and of those born in the UK, 25\% were Pakistani and 25\% were Chinese [36]. In adults, the annual incidence of acute Hep B (based on data from 2002 to 2008, England) has been estimated at 1.3/100,000 person years, with a year-on-year decline [21]. The prevalence of Hep B surface antigen (HBsAg) among the general population in the UK is $<1 \%$ [42]. McLernon et al. [39] estimated the incidence and prevalence of Hep B infection in Tayside, Scotland. In 2003, the prevalence of diagnosed chronic active Hep B was approximately 4/100,000, that of Hep B carriers lay between 26 and 28/100,000 population and that of diagnosed HBsAg+ was around 37/100,000 population [39]. There were no clear trends in incidence, with considerable variation during the included time period of 1989 and 2003 [39].

Another source suggests that the prevalence of Hep B infections may be increasing: Hep B infections among pregnant women in Birmingham increased from 106 cases in 2005 to 161 cases in 2008 ( $p=0.037$ ), with women originating from intermediate and high Hep B prevalence areas of the world having an elevated rate of infection [23]. A number of studies focused on people living in the UK who were born in countries with a high prevalence of Hep $\mathrm{B}$ infection; the data demonstrate that these populations represent a large share of hepatitis infection cases across the UK. Cochrane et al. [26], measured the prevalence of Hep B infection in pregnant women living in Bristol, but born outside the UK, between 2006 and 2014; the overall period prevalence was 1.7\% (95\% CI: 1.4-2.1). Price et al. [44], found that the prevalence of detectable HBsAg in 25,973 patients with HIV in the UK was 6.9\% (6.6-7.2\%). Among 335 African migrants with HIV, the point-prevalence estimate of occult Hep B coinfection was 4.5\% [95\% CI: 2.8-7.4\%] [25]. Similarly, 5.5\% of British-Chinese and South Asian invited volunteers in the North-East of England tested positive for HBsAg (pre-2012) [40]. Estimates from Scotland (2009), suggest that $0.2 \%$ (95\% CI: $0.17-0.24$ ) of the Scottish population aged $\geq 15$ years suffer from chronic hepatitis; East and South Asians represent $44 \%$ of the infected population [46]. A study in Chinese volunteers indicated that they may be subject to care inequalities: 229 volunteers were tested (dried blood spots); HBsAg was positive in $8.7 \%$ of participants $(n=20)$ [52]. Five women and one man were aware of their chronic Hep B infection, but only one man was under specialist care [52]. In an immigration removal center in the south of England, $2 \%$ of detainees were Hep B positive [38]. Tedder et al. [49], recruited 698 patients with chronic Hep B infection from 13 UK hepatology center; $80 \%$ of the patients recruited had been born outside the UK.

As well as people born in countries with a high prevalence of Hep B infection, people who inject drugs (PWID) are also at increased risk of Hep B infection when compared with the general population. Through a survey of 2047 users of psychoactive drugs, Hope et al. [31], found an Hep B core antigen prevalence of 15\% (UK, 2013). Hope et al. [33], who analyzed data from 395 men who had injected image and performance enhancing drugs, found that $9 \%$ had antibodies to the Hep B core antigen (England and Wales, 2011), suggesting a possible increase over time (compared with previous studies). This was confirmed by data from further surveys and the national bio-behavioral surveillance system for PWID, which confirmed an increase in Hep B prevalence in image and performance enhancing drugs injectors over time and showed that it was now similar to that among people injecting psychoactive drugs [32]. In 1999, a Hep B vaccination program was introduced to prisons in Scotland [43]. A survey of 10,000 PWID in Scotland found a positive correlation between Hep B vaccine uptake among PWID and incarceration (adjusted odds ratio: 2.91, 95\% CI: 2.23-3.79) [43], demonstrating the benefits of targeted vaccination programs.

Patients already suffering from a chronic condition appear to be at increased risk of Hep B infection. Of 793 patients on hemodialysis in London, $2 \%$ had chronic Hep B infection, 20\% showed signs of past infection and $42 \%$ had been vaccinated in the past [48], possibly indicating some awareness of the high risk of infection. Among patients with active or latent tuberculosis, recruited in London, $2.6 \%$ were $\mathrm{HBsAg}$ positive, a marker of acute or chronic Hep B infection [41]. HBsAg tests were positive in $0.2 \%$ of 6020 unselected patients attending a genitourinary service [45], showing a higher than average prevalence.

A study evaluating the role of inequalities in infectious diseases did not find a notable connection in the case of Hep B infection [34]. However, this study was performed in the North East of England, and the low prevalence of Hep B in this region may have biased the analysis.

McLernon et al. [39], was the only identified publication on hospital cost data. Hep B patients with HBsAg+ had fewer hospital admissions (and total hospital costs) per patient than immune (i.e., recovered) patients, but a larger percentage of overall hospital costs was due to liver-related admissions (27.6\% for HBsAg+ patients, compared with $14.3 \%$ for immune patients), indicating that the group of immune patients have a higher frequency of comorbid conditions requiring hospitalization. 


\section{Pertussis}

Since the introduction of routine childhood vaccination for pertussis in the 1960s, hospital admission rates for whooping cough have declined in England, from 20 per 100,000 population in 1974 to as low as 1 per 100,000 population in 2010 [30]. The only major rise in incidence was due to a decrease in vaccination uptake around 1980 (47 hospital admissions per 100,000 population in 1982) [30]. In contrast, a 2012 peak in deaths among infants in England cannot be attributed to low vaccination uptake but may be due to the occurrence of pertussis in adolescents with waning immunity [30]. Although the risk is low (0.721 [95\% CI: 0.705-0.763] per 100,000 maternities for all under 1 year-olds; England), pertussis still leads to death in a small number of infants, not yet fully protected by vaccination [51]. To address this emergent pertussis risk to infants, a temporary maternal vaccination program was implemented in September 2012. Based on 2013 data, the effectiveness of maternal pertussis vaccination was found to be $91 \%$ (95\% CI: 84-95) in children under 3 months of age [18]. A later study around the same maternal vaccination program found that preterm infants are less protected by maternal vaccination than full-term infants [22].

Between June 2007 and August 2009, the Health Protection Agency recorded 2443 laboratory-confirmed cases of pertussis in England and Wales [24]. In a UK study, 20\% of children between the ages of five and 15 who presented with persistent cough, were identified as having a recent pertussis infection [53]. The risk for pertussis infection was higher if the preschool booster had been received 7 years or more previously. In adults, pertussis plays a minor role in persistent cough, with 3.3\% of cases due to Bordetella pertussis infection (England and Wales, between 2007 and 2010) [50]. For both teenagers and adults, contact with children between 10 and 14 years has been found to be a risk factor for pertussis (2012 study in England) [54]. The risk of pertussis persists in infants, adolescents and adults in the UK is also confirmed by the data presented by Campbell et al. [6] based on surveillance data from between 1998 and 2009. In infants, the data show that the accelerated vaccination schedule (completed by 4 months of age rather than 6 months of age), introduced in 1990, led to earlier protection [6].

\section{Hemophilus influenzae}

The introduction of the Hib vaccine in 1992 was based on robust clinical data and was carefully planned to ensure supply and offer the required information and training for the public and professionals [55]. After introduction of the Hib vaccine, hospital admissions for meningitis due to Hib decreased drastically and durably, as illustrated by the population-based observational studies by Martin et al. [37], and Collins et al. [28]. The data presented by Martin et al. show that the incidence of $H$. influenzae meningitis hospital admissions in England (annual, per 100,000 children under the age of 15) decreased from 6.72 (95\% CI: 6.18-7.26) in 1992 to 0.39 (95\% CI: 0.26-0.52) in 1994. The annual incidence of invasive Hib disease in England and Wales in children under the age of 5 years decreased from 35.5/100,000 between 1985 and 1990, to only 0.02/100,000 in 2012 [28], although an incidence spike (in the overall population) was observed around 2002/2003 [35]. In spite of the effective disease control with regard to Hib, different types of $H$. influenzae still cause morbidity and mortality in the UK in certain risk groups. For example, $H$. influenzae infection poses a risk to expecting mothers and young infants. Public Health England national surveillance data identified 171 women with laboratory-confirmed invasive $H$. influenzae infection between 2009 and 2012 [29]. Of these cases, 144 (84.2\%; 95\% CI: 77.9-89.3) were due to un-encapsulated (non-typeable $H$. influenzae [NTHi]) types, 11 (6.4\%; 95\% CI: 3.3-11.2) were due to serotype b, and 16 (9.4\%; 95\% CI: 5.4-14.7) were due to other encapsulated serotypes. 75 women were pregnant at the time of infection, indicating a significantly higher infection rate among pregnant women than nonpregnant women $(\mathrm{p}<0.001)$. Infection during the first 24 weeks of pregnancy resulted in fetal loss in $94 \%$ of cases and premature birth in the remaining cases. Infection later during pregnancy led to premature birth in $29 \%$ of cases and stillbirth in $7 \%$ of cases. Collins et al. [27], analyzed invasive Hib infections in neonates ( $<31$ days of age) in England and Wales, between 2000 and 2013. The authors found that the incidence of invasive infections due to NTHi ranged from 2.1 (95\% CI: 1.7-2.5) to 4.8 (95\% CI: 4.3-5.3) per 100,000 live births. Furthermore, although the incidence is low (annual incidence of invasive H. influenzae Type e or Type f [Hie or Hif, respectively] disease in 2010 was 0.090 [95\% CI 0.073-1.10] and 0.030 [95\% CI 0.021-0.042] per 100,000, respectively), a steady increase in invasive Hie and Hif infections has been observed in England and Wales between 2001 and 2010, concerning primarily high-risk groups (children, or adults with comorbidities or immunosuppression) [35]. 


\section{Polio}

Smallman-Raynor et al. [47], modeled the heightened poliomyelitis epidemicity in England and Wales between 1947 and 1957, and concluded that it was associated with an increased rate in the geographical spread of the disease.

\section{Tetanus}

Based on national surveillance data for England from 2001 to 2014 [11], 96 cases of tetanus infection were identified. Only a small percentage of those affected were age-appropriately immunized (8.8\%). Only four cases were reported in children under the age of 16 , with a median age of the affected population of 48 years. These data demonstrate the efficacy of the childhood vaccination program. The data also highlight injecting drug users and not age-appropriately immunized adults sustaining injuries in the home or garden as risk groups.

\section{Diphtheria}

The single identified publication on the incidence of Corynebacterium infections (recorded in England between January 2007 and December 2015) [19], demonstrates the efficacy of the current vaccination schedule. Of the 20 recorded cases, 12 were caused by Corynebacterium ulcerans, likely due to animal contact. In the eight cases of Corynebacterium diphtheriae infection, patients were not fully immunized, and the cases were most likely related to travel.

\section{Discussion}

The results of our systematic literature review demonstrate the continued key role of childhood vaccinations against diphtheria, tetanus, pertussis, Hep B, poliomyelitis and $H$. influenzae invasive disease. Hep B, only very recently added to the UK childhood vaccination schedule (in 2017), reflects the WHO recommendation, as well as increasing concerns for certain risk groups (i.e., people born in countries where Hep B is endemic, their children, PWID, and patients suffering from other chronic conditions). Based on the success of existing vaccination programs [43,56], UK Hep B childhood vaccination may dramatically reduce the risk of Hep B in the general population, and especially in existing risk groups.

Childhood pertussis vaccination has been a great success, significantly and drastically reducing pertussis-related infant mortality. A decrease in vaccination uptake around 1980 led to a major rise in the incidence of pertussis hospital admissions in 1982 [30]. However, the 2012 peak of infant deaths related to pertussis highlights the fact that vaccination programs require periodical updates or modifications. In the specific case of pertussis, a maternal vaccination program has been successful in limiting the number of new, fatal cases of pertussis [18,22]. The source responsible for the 2012 peak has not been clearly identified, although it appears likely that waning immunity in older children and adolescents may be responsible, presenting a reservoir for B. pertussis. Apart from maternal vaccination, which addresses the issue directly, booster vaccination in older children and adults may be an option to limit both infant and adult cases of pertussis [54]. For infants, an accelerated vaccination schedule has proven effective in offering earlier protection [6].

Hib disease is well-controlled by the UK childhood vaccination program (in place since 1992). The remaining low number of cases in risk groups (i.e. pregnant women, where fetal loss occurs in $94 \%$ of cases [29], young infants, and adults with comorbidities or immunosuppression) [35] is largely due to unencapsulated/NTHi and other non-b capsular types/serotypes (i.e., Hie or Hif), although Hib vaccination provides some cross-protection against Hif or Hie infection. The exploration of ways to increase cross-protection in vaccines or the use of vaccines against $H$. influenzae types other than b could prove valuable.

Polio is close to eradication, with only pockets of the disease remaining globally. Europe has been declared polio-free in 2002 [57]. In the UK, the last confirmed case of indigenous wild polio was recorded in the 1980s, after introduction of IPV in the 1950s [13]. Therefore, only a single publication, a historical overview [47], was identified by our review. However, isolated cases of imported polio virus may still occur, and childhood vaccination should be maintained until complete global polio virus eradication, with reservoirs in Afghanistan and Pakistan remaining a threat [58].

Thanks to the tetanus childhood vaccination course (and on-demand booster vaccination) [59], the incidence of tetanus in the UK is very low, mostly limited to risk groups, which include PWID and adults sustaining physical injuries [11]. 
The available evidence shows clearly that diphtheria is very well controlled by the current childhood vaccination program [19], However, since diphtheria can still be contracted by animal contact or travel, continued childhood vaccination remains important.

Our analysis provides a systematic overview of the available evidence around the clinical burden of diphtheria, tetanus, pertussis, Hep B, poliomyelitis and H. influenzae invasive disease in the UK. However, it was limited by the fact that the publication date had to be restricted to 2012 and onwards (most relevant to the burden), due to the large number of identified studies. Our review also only identified a single study relating to cost burden [39], highlighting a gap in recent publications in this field, most likely indicating that the cost burden of the diseases evaluated is small. Furthermore, outcomes and outcome measures differed between publications, making it difficult to synthesize the available results for any single disease area or population.

Vaccinations, vaccination schedules, and vaccination programs will have to continue to adapt and change in order to keep pace with emerging threats and changes in the vaccine-preventable disease landscape. Vaccination remains the pre-eminent measure to prevent diphtheria, tetanus, pertussis, Hep B, poliomyelitis and H. influenzae invasive disease in the UK. The importance of population-wide adherence to childhood vaccination programs is exemplified by repeated measles outbreaks across Europe, due to suboptimal vaccination coverage [60,61], and this highlights the criticality of investing in vaccination and vaccine confidence to protect public health. It is crucial that high vaccination coverage rates are maintained on a population level to minimize the risk of such outbreaks becoming commonplace in the future.

\section{Conclusion}

Thanks to a comprehensive childhood vaccination program, diphtheria, tetanus, pertussis, Hep B, poliomyelitis and $H$. influenzae invasive disease are currently well controlled in the UK. The available data suggest that their clinical burden is low, although certain population subgroups are at higher risk than the general population. Any emerging peaks in incidence are being addressed promptly by preventive counter measures. High vaccine coverage rates remain essential in maintaining population health. The COVID-19 pandemic, as well as the data identified by our review, has also illustrated the need for global coordination and collaboration in the control of infectious diseases and vaccinations.

\section{Future perspective}

Below target coverage rates for routine childhood vaccinations are a growing concern globally. Adhering to vaccination coverage targets is critical to eliminate a disease or prevent outbreaks. This means increasing investment in vaccination and awareness campaigns to protect public health globally. In developed countries, concerns around adverse events associated with childhood vaccinations have contributed to lowering vaccination coverage, as well as immigration from countries where certain diseases are endemic and childhood vaccination programs less or only recently established. Most recently, the restrictions imposed on populations as a result of the COVID-19 pandemic have led to a decrease in the uptake of childhood vaccinations, increasing the risk of future outbreaks of diseases that are currently well controlled. Continued investment in disease prevention through vaccination is imperative to ensure public health is protected in the future.

One method that could help to address low coverage rate is the use of combination vaccines. Combination vaccines are already widely used to decrease injection burden, and further innovations may offer additional benefits [5].

The changing disease landscape will require continued adaptation and optimization of the childhood vaccination program. The addition of Hep B vaccination to the UK childhood vaccination is an example of a recent adaptation of the UK's vaccination program. The incidence and prevalence data for $H$. influenzae invasive disease, showing that most recorded cases are due to serotypes other than Hib, may indicate an emerging threat, which may have to be addressed by a modified vaccination strategy.

\section{Supplementary data}

To view the supplementary data that accompany this paper please visit the journal website at: www.futuremedicine.com/doi/sup $\mathrm{pl} / 10.2217 / \mathrm{fmb}-2020-0170$

\section{Author contributions}

All authors collaborated in the design of the systematic review. C McCloskey and P Hudson performed the systematic review. All authors contributed to the manuscript, and reviewed and agreed on the content. 
Financial \& competing interests disclosure

This analysis was sponsored by Sanofi. J Clark-Wright and S Carroll are employees of Sanofi. P Hudson and C McCloskey are employees of DRG Abacus. The authors have no other relevant affiliations or financial involvement with any organization or entity with a financial interest in or financial conflict with the subject matter or materials discussed in the manuscript apart from those disclosed.

Writing assistance was provided by Brigitte Moore, DRG Abacus, funded by Sanofi.

\section{Open access}

This work is licensed under the Attribution-NonCommercial-NoDerivatives 4.0 Unported License. To view a copy of this license, visit http://creativecommons.org/licenses/by-nc-nd/4.0/

\section{Executive summary}

Disease burden of diphtheria, tetanus, pertussis, hepatitis B, poliomyelitis \& Hemophilus influenzae invasive disease in the UK

- Hepatitis B (Hep B) vaccination was introduced in 2017, to address concerns around risk groups (e.g., people born in countries where Hep B is endemic or people who inject drugs).

- Although the incidence of pertussis is low, reservoirs remain in the population.

- Cases of $H$. influenzae infection are primarily due to strains not covered by the standard Hib vaccine.

- There have been no cases of polio in the UK, but vaccination remains crucial in view of the reservoirs in Afghanistan and Pakistan.

- The incidence of tetanus and diphtheria is low and restricted to risk groups.

Importance of childhood vaccination programs

- Continued childhood vaccination programs, achieving sufficient vaccination coverage, are crucial in preventing outbreaks and incidence peaks of preventable diseases.

\section{References}

Papers of special note have been highlighted as: $\bullet$ of interest

1. Ehreth J. The global value of vaccination. Vaccine 21(7-8), 596-600 (2003).

2. Public Health England. Vaccination timeline (2017). https://assets.publishing.service.gov.uk/government/uploads/system/uploads/attachment_data/file/650485/Vaccination_timeline.pdf

3. Public Health England. Diphtheria: notifications and deaths, England and Wales. Diphtheria: notifications cases and deaths, England and Wales, 1914 onward. (2014). https://assets.publishing.service.gov.uk/government/uploads/system/uploads/attachment_data/file/45 0155/Diphtheria_notifications_deaths_Eng_Wales_1914_onward.pdf

4. Choi YH, Campbell H, Amirthalingam G, Van Hoek AJ, Miller E. Investigating the pertussis resurgence in England and Wales, and options for future control. BMC Medicine 14(1), 121 (2016).

5. Maman K, Zöllner Y, Greco D, Duru G, Sendyona S, Remy V. The value of childhood combination vaccines: from beliefs to evidence. Hum. Vaccin. Immunother. 11(9), 2132-2141 (2015).

6. Campbell H, Amirthalingam G, Andrews N et al. Accelerating control of pertussis in England and Wales. Emerg. Infect. Dis. 18(1), 38-47 (2012).

7. Zhang L, Prietsch SO, Axelsson I, Halperin SA. Acellular vaccines for preventing whooping cough in children. Cochrane Database Syst. Rev. Cd001478 (2014) (Epub ahead of print).

8. Gambhir M, Clark TA, Cauchemez S, Tartof SY, Swerdlow DL, Ferguson NM. A change in vaccine efficacy and duration of protection explains recent rises in pertussis incidence in the United States. PLoS Comput. Biol. 11(4), e1004138 (2015).

9. Heath PT, Mcvernon J. The UK Hib vaccine experience. Arch. Dis. Child. 86(6), 396-399 (2002).

10. Public Health England. Laboratory reports of Haemophilus influenzae by age group and serotype (England and Wales): October to December 2015, and consolidated annual report for 2015. Health Protection Report 10(8),

(2016). https://assets.publishing.service.gov.uk/government/uploads/system/uploads/attachment_data/file/503792/hpr0816_hib.pdf

11. Collins S, Amirthalingam G, Beeching NJ et al. Current epidemiology of tetanus in England, 2001-2014. Epidemiol. Infect. 144(16), 3343-3353 (2016).

12. Public Health England. Research and analysis. Tetanus is England and Wales: 2013 (2013). www.gov.uk/government/publications/tetanus-in-england-and-wales-2013/tetanus-in-england-and-wales-2013

13. Salisbury DM, Brown DW, White JM, Ramsay ME. Polio eradication: surveillance implications for the United Kingdom. J. Infect. Dis. 175(Suppl. 1), S156-S159 (1997). 
14. Polio Global Eradiation Initiative. Endemic countries (2018). http://polioeradication.org/where-we-work/polio-endemic-countries/

15. National Health Service. Childhood vaccination coverage statistics, England, 2017-18. Report. https://files.digital.nhs.uk/55/D9C4C2/child-vacc-stat-eng-2017-18-report.pdf Last accessed 25 February 2019

16. National Health Service. Childhood vaccination coverage statistics, England, 2016-17. Childhood Vaccination Coverage Statistics 2016-17: Summary Infographic. (2017). https://digital.nhs.uk/data-and-information/publications/statistical/childhood-vaccination-cov erage-statistics/childhood-vaccination-coverage-statistics-england-2016-17

17. Drummond MF, Jefferson TO. Guidelines for authors and peer reviewers of economic submissions to the BMJ. The BMJ Economic Evaluation Working Party. BMJ (Clinical Research Ed.) 313(7052), 275-283 (1996).

18. Amirthalingam G, Andrews N, Campbell $\mathrm{H}$ et al. Effectiveness of maternal pertussis vaccination in England: an observational study. Lancet 384(9953), 1521-1528 (2014).

19. Both L, Collins S, De Zoysa A, White J, Mandal S, Efstratiou A. Molecular and epidemiological review of toxigenic diphtheria infections in England between 2007 and 2013. J. Clin. Microbiol. 53(2), 567-572 (2015).

20. Braccio S, Irwin A, Riordan A et al. Acute infectious hepatitis in hospitalised children: A British Paediatric Surveillance Unit study. Arch. Dis. Child. 102(7), 624-628 (2017).

21. Brant LJ, Hurrelle M, Collins S, Klapper PE, Ramsay ME. Using automated extraction of hepatitis B tests for surveillance: evidence of decreasing incidence of acute hepatitis B in England. Epidemiol. Infect. 140(6), 1075-1086 (2012).

22. Byrne L, Campbell H, Andrews N, Ribeiro S, Amirthalingam G. Hospitalisation of preterm infants with pertussis in the context of a maternal vaccination programme in England. Arch. Dis. Child. 16, 16 (2017).

23. Caley M, Fowler T, Greatrex S, Wood A. Differences in hepatitis B infection rate between ethnic groups in antenatal women in Birmingham, United Kingdom, May 2004 to December 2008. Eurosurveillance 17(30), 20120726 (2012).

- Highlights the possibility of increased hepatitis B (Hep B) infections among pregnant women.

24. Campbell H, Amirthalingam G, Fry NK et al. Oral fluid testing for pertussis, England and Wales, June 2007-August 2009. Emerg. Infect. Dis. 20(6), 968-975 (2014).

25. Chadwick D, Doyle T, Ellis S et al. Occult hepatitis B/HIV co-infection in African migrants to the UK: a point prevalence study. HIV Medicine 14, 3 (2013).

26. Cochrane A, Evlampidou I, Irish C, Ingle SM, Hickman M. Hepatitis B infection prevalence by country of birth in migrant populations in a large UK city. J. Clin, Virol. 68, 79-82 (2015).

27. Collins S, Litt DJ, Flynn S, Ramsay ME, Slack MP, Ladhani SN. Neonatal invasive Haemophilus influenzae disease in England and Wales: epidemiology, clinical characteristics, and outcome. Clin. Infect. Dis. 60(12), 1786-1792 (2015).

28. Collins S, Ramsay M, Campbell H, Slack MP, Ladhani SN. Invasive Haemophilus influenzae Type b disease in England and Wales: who is at risk after 2 decades of routine childhood vaccination? Clin. Infect. Dis. 57(12), 1715-1721 (2013).

29. Collins S, Ramsay M, Slack MP et al. Risk of invasive Haemophilus influenzae infection during pregnancy and association with adverse fetal outcomes. JAMA 311(11), 1125-1132 (2014).

- Highlights the possible relevence of non-Hib Hemophilus influenzae infections.

30. Haslam N, Hoang U, Goldacre MJ. Trends in hospital admission rates for whooping cough in England across five decades: database studies. J. R. Soc. Med. 107(4), 157-162 (2014).

31. Hope VD, Cullen KJ, Smith J, Jessop L, Parry J, Ncube F. Is the recent emergence of mephedrone injecting in the United Kingdom associated with elevated risk behaviours and blood borne virus infection? Eurosurveillance 21(19), 30225 (2016)

- Discusses the prevalence of Hep B in people who inject drugs.

32. Hope VD, Harris R, Mcveigh J et al. Risk of HIV and hepatitis B and C over time among men who inject image and performance enhancing drugs in England and Wales: results from cross-sectional prevalence surveys, 1992-2013. J. Acquir. Immune Defic. Syndr. 71(3), 331-337 (2016b).

33. Hope VD, Mcveigh J, Marongiu A et al. Prevalence of, and risk factors for, HIV, hepatitis B and C infections among men who inject image and performance enhancing drugs: a cross-sectional study. BMJ Open 3(9), e003207 (2013).

34. Hughes GJ, Gorton R. Inequalities in the incidence of infectious disease in the North East of England: a population-based study. Epidemiol. Infect. 143(1), 189-201 (2015).

35. Ladhani SN, Collins S, Vickers A et al. Invasive Haemophilus influenzae serotype e and $\mathrm{f}$ disease, England and Wales. Emerg. Infect. Dis. 18(5), 725-732 (2012).

- Highlights the possible relevence of non-Hib $H$. influenzae infections.

36. Ladhani SN, Flood JS, Amirthalingam G et al. Epidemiology and clinical features of childhood chronic hepatitis B infection diagnosed in England. Pediatr. Infect. Dis. J. 33(2), 130-135 (2014).

- Children from minority backgrounds are at higher than average risk of chronic Hep B. 
37. Martin NG, Sadarangani M, Pollard AJ, Goldacre MJ. Hospital admission rates for meningitis and septicaemia caused by Haemophilus influenzae, Neisseria meningitidis, and Streptococcus pneumoniae in children in England over five decades: a population-based observational study. Lancet Infect. Dis. 14(5), 397-405 (2014).

38. Mclaren E, Baugh V, Plugge E, O'Moore E. Describing the burden of infectious diseases among a population of detainees in an immigration removal centre (IRC) in the United Kingdom: a descriptive epidemiological approach. J. Immigr. Minor. Health 15(4), 764-770 (2013).

39. Mclernon DJ, Donnan PT, Dillon JF. Prevalence estimates of diagnosed viral hepatitis B, liver condition outcomes and hospitalization costs: a population record-linkage study in Tayside, Scotland. Epidemiol. Infect. 141(10), 2122-2130 (2013).

40. Mcpherson S, Valappil M, Moses SE et al. Targeted case finding for hepatitis B using dry blood spot testing in the British-Chinese and South Asian populations of the North-East of England. J. Viral Hepat. 20(9), 638-644 (2013).

41. Nooredinvand HA, Connell DW, Asgheddi M et al. Viral hepatitis prevalence in patients with active and latent tuberculosis. World J. Gastroenterol. 21(29), 8974-8980 (2015).

42. Ozaras R, Corti G, Ruta S et al. Differences in the availability of diagnostics and treatment modalities for chronic hepatitis B across Europe. Clin. Microbiol. Infect. 21(11), 1027-1032 (2015).

43. Palmateer NE, Goldberg DJ, Munro A et al. Association between universal hepatitis B prison vaccination, vaccine uptake and hepatitis B infection among people who inject drugs. Addiction 15, 15 (2017).

44. Price H, Bansi L, Sabin CA et al. Hepatitis B virus infection in HIV-positive individuals in the UK collaborative HIV cohort (UK CHIC) study. PLoS ONE 7(11), e49314 (2012).

45. Price H, Salimee S, Coelho D. Prevalence of hepatitis B and hepatitis C in a UK genitourinary medicine clinic. Int. J. STD AIDS 28(3), 238-241 (2017).

46. Schnier C, Wallace L, Tempelton K et al. Use of laboratory-based surveillance data to estimate the number of people chronically infected with hepatitis B living in Scotland. Epidemiol. Infect. 142(10), 2121-2130 (2014).

47. Smallman-Raynor MR, Cliff AD. Abrupt transition to heightened poliomyelitis epidemicity in England and Wales, 1947-1957, associated with a pronounced increase in the geographical rate of disease propagation. Epidemiol. Infect. 142(3), 577-591 (2013).

48. Sowole L, Labbett W, Patel M et al. The prevalence of occult hepatitis B virus (hbv) infection in a large multi-ethnic haemodialysis cohort. BMC Nephrol. 16(1), 12 (2015).

49. Tedder RS, Rodger AJ, Fries L et al. The diversity and management of chronic hepatitis B virus infections in the United Kingdom: a wake-up call. Clin. Infect. Dis. 56(7), 951-960 (2013).

50. Teepe J, Broekhuizen BDL, Kretzschmar M et al. Prevalence, diagnosis, and disease course of pertussis in adults with acute cough: a prospective, observational study in primary care. Br. J. Gen. Pract. 65(639), e662-e667 (2015).

51. Van Hoek AJ, Campbell H, Amirthalingam G, Andrews N, Miller E. The number of deaths among infants under one year of age in England with pertussis: results of a capture/recapture analysis for the period 2001 to 2011. Eurosurveillance 18(9),

(2013). www.eurosurveillance.org/images/dynamic/EE/V18N09/art20414.pdf

52. Vedio AB, Ellam H, Rayner F et al. Hepatitis B: report of prevalence and access to healthcare among Chinese residents in Sheffield UK. J. Infect. Public Health 6(6), 448-455 (2013).

\section{- Care inequalities may exist in certain populations.}

53. Wang K, Fry NK, Campbell $\mathrm{H}$ et al. Whooping cough in school age children presenting with persistent cough in UK primary care after introduction of the preschool pertussis booster vaccination: prospective cohort study. BMJ (Clinical Research ed.) 348, g3668 (2014).

54. Wensley A, Hughes GJ, Campbell $\mathrm{H}$ et al. Risk factors for pertussis in adults and teenagers in England. Epidemiol. Infect. 145(5), 1025-1036 (2017).

55. Salisbury DM. The introduction of Haemophilus influenzae Type b immunization into the United Kingdom: practical steps to assure success. Pediatr. Infect. Dis. J. 17(Suppl. 9), S136-S139 (1998).

56. Meireles LC, Marinho RT, Van Damme P. Three decades of hepatitis B control with vaccination. World J. Hepatol. 7(18), 2127-2132 (2015).

57. Unicef. Europe achieves historic milestone as region is declared polio-free. Joint Press Release (2002). www.unicef.org/newsline/02pr40polio.htm

58. World Health Organization. Maintaining polio-free status of European Region as part of the global polio Endgame Strategy (2018). www.euro.who.int/en/health-topics/communicable-diseases/poliomyelitis/maintaining-polio-free-status-of-europeanregion-as-part-of-the-global-polio-endgame-strategy

59. National Health Service. Tetanus vaccination (2017). www.nhs.uk/conditions/tetanus/\#tetanus-vaccination

60. Muscat M. Who gets measles in Europe? J. Infect. Dis. 204(Suppl. 1), S353-S365 (2011).

61. Carrillo-Santisteve P, Lopalco PL. Measles still spreads in Europe: who is responsible for the failure to vaccinate? Clin. Microbiol. Infect. 18(Suppl. 5), 50-56 (2012). 